\title{
GAIA Insufficient Evidence for Diagnosis of Preeclampsia
}

National Cancer Institute

\section{Source}

National Cancer Institute. GAIA Insufficient Evidence for Diagnosis of Preeclampsia. NCI

Thesaurus. Code C128005.

GAIA Insufficient Evidence for Diagnosis of Preeclampsia is defined as a pregnancy with gestational age greater than or equal to 20 weeks and either a) The inability to measure blood pressure OR b) The inability to evaluate for proteinuria. 